\title{
Evaluación de la percepción de pacientes sobre el comportamiento de comunicación del personal de enfermería: diseño y validación en población mexicana
}

\author{
Cristina Müggenburg, ${ }^{1}$ Rebeca Robles, ${ }^{2}$ Alejandra Valencia, ${ }^{3}$ María del Carmen Hernández Guillén, ${ }^{1}$ Sandra \\ Olvera, ${ }^{4}$ Angélica Riveros Rosas ${ }^{5}$
}

Artículo original

\section{ABSTRACT \\ Background}

Nurse-patient communication has been established as a key component of professional healthcare. The present study examined the patients' perception of the verbal and non-verbal communication skills of nurses. Although the Caring Assessment Tool (CAT) stands among the instruments available to evaluate these communication skills, cultural and schooling characteristics limit its direct applicability to the Mexican context.

\section{Objective}

The aim of this study was to design and validate an instrument on the patient-recorded Nurse Communication Behavior (CECOP, for its acronym in Spanish) based on the Mexican patient's experience.

\section{Method}

Based on exploratory interviews with 29 patients, a first draft of the CECOP was designed. The instrument was validated by independent expert judges and after the elimination of two items it was used with 150 patients. The validity was assessed by a factor analysis extraction through Principal Component Analysis and Varimax Rotation. Convergence validity was established between the CAT and the CECOP and between the CECOP and numeric scales that evaluate empathy and patient satisfaction.

\section{Results}

Construct Validity reduced the CECOP to 10 items in two factors (empathy and respect) with statistically significant KMO (Kaiser-Meyer-Olkin) and Bartlett's Test ( $p \leq .001)$. The explained variation was $54.58 \%$. The correlation between the CECOP score (10 items) and the CAT was .459. Between the CECOP and the empathy scale and between the CECOP and the satisfaction scale the correlations were .419 and .495 , respectively. All correlations were statistically significant $(p \leq .001)$.

\section{Discussion and conclusion}

The CECOP can be used to evaluate professional care in its human dimension from the patient's perspective and to suggest improvements in the nurses communication.

Key words: Nurse-patient communication, perception by patients, nurse-patient relation.

\section{RESUMEN}

\section{Antecedentes}

La comunicación enfermera-paciente es fundamental para la aplicación de los cuidados en salud. El presente estudio aborda la percepción de pacientes sobre la comunicación verbal y no verbal con sus enfermeras. Entre los instrumentos para su evaluación destaca la Herramienta para la Evaluación del Cuidado (CAT por sus siglas en inglés), pero diferencias culturales y educativas de la población mexicana limitan su aplicabilidad en nuestro contexto.

\section{Objetivo}

Diseñar y validar un instrumento sobre la percepción de los pacientes sobre el comportamiento de comunicación de enfermeras (denominado CECOP), en función de lo que observan pacientes mexicanos.

\section{Método}

Con base en una entrevista exploratoria a 29 pacientes, se diseñó el CECOP con 25 reactivos, se estableció validez de contenido con jueces expertos (eliminando dos reactivos) y se aplicó a 150 pacientes. Se empleó análisis factorial con método de extracción de análisis de componentes principales y rotación Varimax. Se estableció la validez convergente entre el CECOP y el CAT y entre el CECOP y escalas numéricas que valoraron comprensión empática y satisfacción del paciente.

Coordinación de Investigación, Escuela Nacional de Enfermería y Obstetricia, Universidad Nacional Autónoma de México.

2 Dirección de Investigaciones Epidemiológicas y Psicosociales, Instituto Nacional de Psiquiatría Ramón de la Fuente Muñiz.

3 División de Investigación y Posgrado, Facultad de Psicología, Universidad Nacional Autónoma de México.

4 Coordinación de Investigación, Instituto Nacional de Cardiología Ignacio Chávez.

División de Investigación, Facultad de Contaduría y Administración, Universidad Nacional Autónoma de México.

Correspondencia: Cristina Müggenburg Rodríguez Vigil. Río Mixcoac 229-2, Florida, Álvaro Obregón, 01030, México DF. E-mail: cmuggenb@yahoo.com.mx Recibido primera versión: 8 de enero de 2014. Segunda versión: 4 de diciembre de 2014. Aceptado: 12 de marzo de 2015. 


\section{Resultados}

La validez de constructo lo redujo a diez reactivos en dos factores (empatía y respeto); con Kaiser-Meyer-Olkin (KMO) y prueba de Bartlett estadísticamente significativas $(\mathrm{p} \leq .001)$. La varianza explicada fue de $54.58 \%$. La correlación entre los puntajes totales del CECOP (10 reactivos) y el CAT fue .459 , el CECOP y la escala de comprensión fue .419; el CECOP y la escala de satisfacción, .495; todas, estadísticamente significativas ( $\mathrm{p} \leq .001)$.
Discusión y conclusión

El CECOP permite evaluar los cuidados profesionales en su dimensión humana, desde la percepción de los pacientes, y sugerir propuestas para mejorar la comunicación de las enfermeras.

Palabras clave: Comunicación enfermera-paciente, percepción del paciente, relaciones enfermera-paciente.

\section{ANTECEDENTES}

La comunicación enfermera-paciente es el eje articulador de los cuidados de enfermería. También es el medio para conocer al enfermo y comprenderlo, así como una fuente de información para procurar cuidados técnicos individualizados que favorezcan la comprensión y aceptación de los mismos por parte de los pacientes. ${ }^{1}$

La comunicación de las enfermeras ${ }^{a}$ se ha investigado a partir de estudios descriptivos sobre los comportamientos de interacción personal de ellas con sus pacientes. Los resultados muestran que el cuidado implica cercanía con el paciente por medio de expresiones verbales y no verbales que generan comunicación que favorece la aplicación de cuidados técnicos y propicia la satisfacción del paciente y de la enfermera como resultado de la interacción..$^{2-6}$ Otros estudios se han enfocado en el diseño y validación de instrumentos sobre la interacción enfermera-paciente mediante la construcción y evaluación de indicadores del cuidado de la persona. Asimismo, han procurado su validación de contenido y de constructo, así como medir la satisfacción del paciente; indicador de la calidad de la atención de las enfermeras. ${ }^{7-13}$

Adicionalmente, se han analizado los cambios derivados de entrenamientos dirigidos a mejorar la comunicación de las enfermeras con sus pacientes, en variables tales como la actitud de las enfermeras, el bienestar de ambos, la percepción de los pacientes en cuanto a la empatía de las enfermeras, la sensación de confianza, la disminución del estrés y el aumento de la satisfacción de los pacientes con la atención. Sin embargo, no en todos los casos se han logrado diferencias estadísticamente significativas. ${ }^{14-19}$ En México, los estudios son muy escasos y se circunscriben al indicador de calidad trato digno, ${ }^{20,21}$ con excepción de un artículo que ha reportado que el entrenamiento en interacción enfermera-paciente repercute en el bienestar percibido por el enfermo y en algunos indicadores de recuperación clínica. ${ }^{15}$

El efecto terapéutico que tiene la comunicación de la enfermera que asiste al paciente hospitalizado, ${ }^{22-26}$ así como la

a En este artículo se emplea el término enfermera o enfermeras por convención, por ser un grupo mayoritariamente de género femenino, en ningún momento se pretende excluir a los profesionales de enfermería de género masculino. posibilidad de desarrollar habilidades de comunicación en programas de actualización para las enfermeras, ${ }^{22,23,26,27}$ sustentan la necesidad de contar con mejores indicadores de las habilidades de comunicación que incidan en la recuperación de los pacientes y en la calidad percibida de los servicios de salud.

En una primera etapa del presente estudio se buscaron instrumentos que midieran la interacción enfermera-paciente, que fueran lo suficientemente sensibles para evaluar intervenciones en habilidades de comunicación. Inicialmente se seleccionó un instrumento diseñado por Duffy, ${ }^{9,10,27}$ constituido por 36 reactivos con ocho factores, cuatro de ellos relacionados con empatía y uno con respeto. Sin embargo, durante el piloteo se observaron dificultades en la comprensión de los reactivos, muy probablemente atribuibles a diferencias culturales, al nivel educativo o a concepciones derivadas del lenguaje verbal y no verbal en el contexto mexicano. Por otra parte, la extensión de dicho instrumento también pudo ser un factor promotor de cansancio entre los participantes. Lo anterior llevó a la necesidad de diseñar y validar un instrumento diseñado sobre los comportamientos de las enfermeras relacionados con su forma de comunicación observada por los pacientes (CECOP) en el contexto cultural mexicano.

La creación de un catálogo de comportamientos, descrito por López y Torres, ${ }^{28}$ ofreció elementos que guiaron la elaboración de este instrumento. Los autores propusieron un procedimiento sistemático en el diseño de este tipo de instrumentos, además de advertir la dificultad para identificar y definir las categorías de los comportamientos, la definición de constructos y sus componentes, la definición de la muestra de estudio en el marco de la población de referencia, la determinación de su tamaño, el registro narrativo del comportamiento y las características de sus episodios y sus escenarios.

En el presente estudio se consideraron los lineamientos de procedimiento referidos por los autores para obtener las categorías mediante entrevista a los pacientes, en virtud de la imposibilidad de hacer observaciones directas sobre la interacción enfermera-paciente en las instituciones participantes.

En México, la interacción enfermera-paciente ha sido medida mediante el indicador de trato digno ${ }^{20,21}$ propues- 
to en la evaluación de la calidad de los servicios de salud pública de la Secretaría de Salud, ${ }^{29}$ mismo que puede ser complementado con otros indicadores para identificar habilidades específicas con capacidad de entrenamiento para la mejora de la calidad de los servicios en cuanto a la interacción personal. Por tanto, el diseño de un instrumento que brinde información para la operación de estrategias que incrementen la calidad de los servicios puede ser un aporte útil para las instituciones hospitalarias mexicanas.

El constructo que motiva el diseño y validación de este instrumento se fundamenta en los siguientes sustentos teóricos: 1. La comunicación interpersonal tiene dos formas de expresión: comunicación verbal y comunicación no verbal. 24,30,31 2 . La comunicación o interacción entre la enfermera y el paciente tiene un efecto terapéutico. ${ }^{22-26} 3$. La empatía y el respeto son condiciones facilitadoras de la relación terapéutica y colaboración del paciente. La primera se define como la capacidad de comprender correctamente lo que experimenta la otra persona, no sólo entender las palabras, sino los sentimientos y las emociones que hay detrás de éstas, valorar con precisión sus preocupaciones y problemas, obteniendo así su confianza. En tanto, el respeto implica aceptación positiva, manifestar disposición de tiempo y energía, valorar al paciente como persona única con las cualidades y los recursos que pueden estar bloqueados por la enfermedad, por la educación o que no han sido estimulados para ser ejercidos. ${ }^{24,30}$ Por último, 4 . La escucha y la comunicación eficaz son habilidades fundamentales para una buena relación terapéutica. ${ }^{32}$

El uso del constructo "comunicación enfermera-paciente" se basa en el efecto terapéutico de la interacción que ocurre cuando la enfermera cuida o atiende al paciente hospitalizado, ${ }^{24,26}$ el cual sucede fuera del entorno habitual del paciente. ${ }^{33,34}$ La selección de los subconjuntos de análisis o componentes "empatía" y "respeto", se funda en la observación que realizan los pacientes sobre las formas de comunicación verbal y no verbal de las enfermeras que los atienden. La relevancia es la que asigna el paciente durante su estancia hospitalaria, pues se busca definir conductas que puedan ser objeto específico de entrenamiento.

\section{MÉTODO}

Con base en los lineamientos del catálogo de López y Torres, ${ }^{28}$ así como en los supuestos teóricos relacionados con el proceso de comunicación enfermera-paciente, se inició el diseño de un instrumento de comportamiento de las enfermeras sobre su forma de comunicación con el paciente hospitalizado.

Se elaboró una guía para una entrevista semiestructurada a pacientes que reunían los siguientes criterios: adultos, conscientes, orientados, sin alteraciones de conducta, con padecimientos crónico-degenerativos, hospitalizados en una institución de tercer nivel del sector público del área urbana de la Ciudad de México, la cual recibe usuarios, no derechohabientes de procedencia local y nacional. La guía se enfocó en las condiciones facilitadoras de la relación terapéutica: empatía y respeto, ${ }^{30}$ examinadas a partir de las formas de comunicación verbal y no verbal. Quedó integrada por cinco apartados:

1. El primer apartado exploró de forma general la percepción del paciente en cuanto a la empatía y el respeto que recibe por parte de las enfermeras que lo atienden. Se elaboraron cuatro preguntas: las primeras solicitan una respuesta binaria referida a la presencia o ausencia de dicho comportamiento y las restantes evalúan la calidad atribuida al comportamiento por medio de una escala análoga visual. Las preguntas fueron: ¿Se siente comprendido por las enfermeras que lo atienden? Con opción de respuesta sí o no; ¿Cómo califica la comprensión que recibe?

Con opción de respuesta de uno a 10 (de menos a más); ¿Se siente respetado por las enfermeras que lo atienden? Con opción de respuesta sí o no, y ¿Cómo califica el respeto que le tienen? Con opción de respuesta de uno a 10 (de menos a más).

2. El segundo correspondió a preguntas abiertas: ¿Qué le hace pensar que la enfermera lo comprende?, ¿Qué conductas favorables observa en ella?, ¿Qué le hace pensar que la enfermera no lo comprende?, ¿Qué conductas desfavorables observa en ella?, ¿Qué le hace pensar que la enfermera lo respeta?, ¿Qué conductas favorables observa en ella?, ¿Qué le hace pensar que la enfermera no lo respeta? y ¿Qué conductas desfavorables observa en ella?

3. Previendo que la exploración no fluyera en forma natural, se elaboró un listado con formas de comunicación verbal y no verbal involucradas en la empatía y el respeto, incluyendo contacto visual, contacto físico, posición corporal, movimientos corporales, tono de voz, tipo de vocabulario, momentos de silencio durante la conversación, palabras de apoyo, expresiones faciales, frases de elogio, actitud de escucha, respuesta a preguntas formuladas por el paciente e información sobre la enfermedad.

Con el fin de facilitar al paciente la identificación y recordatorio de los aspectos comportamentales de la atención que recibe, las formas de comunicación se representaron a manera de ejemplo para permitir al paciente ubicar conductas observables y así dar lugar a la expresión particular de aquello que relacionaban con el contenido de la pregunta abierta. Para evitar una inducción o sesgo de las respuestas se puso especial cuidado en que los ejemplos se expresaran en forma neutral, sin implicaciones positivas o negativas, sobre los comportamientos. En todos los casos se anotaron las respuestas textuales emitidas por los pacientes. 
4. El cuarto apartado ofreció la oportunidad al paciente para comentar algo que pudiera recomendar a las enfermeras para mejorar la atención o cuidado de los pacientes hospitalizados, con el fin de recabar información que pudiera retroalimentar aspectos comportamentales relacionados con la comunicación interpersonal.

5. Por último, se recolectaron datos sociodemográficos como edad, género, estado civil y escolaridad, a fin de caracterizar a la población objeto de esta exploración diagnóstica.

\section{Procedimiento}

Con base en la guía, previa evaluación del Comité de Investigación y Ética de la Institución hospitalaria seleccionada y con el consentimiento de los pacientes (de acuerdo con el Reglamento de la Ley General de Salud en materia de investigación para la salud, en el Título Segundo de los Aspectos Éticos para la investigación en seres humanos ${ }^{17,20,23}$ se realizaron 16 entrevistas a profundidad a ocho pacientes adultos de sexo masculino y ocho del femenino. El tamaño de la muestra, de acuerdo con el propósito de la aplicación, respondió al criterio de saturación de los hallazgos repetidos. Es decir, cuando la información de las entrevistas ya no aportaba información diferente. ${ }^{35} \mathrm{El}$ tiempo empleado por entrevista fue de entre 30 y 45 minutos.

\section{Validación}

Se categorizaron las respuestas en función de su contenido y se convirtieron en reactivos. Los reactivos obtenidos se sometieron a una segunda prueba piloto con 13 pacientes más de la misma institución hospitalaria, a fin de verificar su claridad, realizar los ajustes necesarios y favorecer la confianza de los pacientes.

El instrumento obtenido fue sometido a juicio de seis expertos con el fin de establecer la validez de contenido por reactivo (VCR) y la validez de contenido general (VCG) de acuerdo con Lawshe ${ }^{36}$ y Tristán. ${ }^{37} \mathrm{El}$ análisis y valoración lo realizaron seis expertas que reunían los siguientes criterios: especialistas en psicología con enfoque en terapia cognitivo conductual (TCC) y entrenamiento en habilidades sociales, enfermeras o con experiencia con enfermeras.

Para la validación de constructo se procedió a la prueba piloto del instrumento en 150 pacientes adultos, conscientes, orientados, sin alteraciones de conducta, con padecimientos crónico-degenerativos, hospitalizados en una institución de tercer nivel del sector público, similar a la de la exploración inicial. Simultáneamente se aplicó el instrumento de Duffy ${ }^{9}$ denominado CAT (por sus siglas en inglés) con consistencia teórica y empírica, estructurado con ocho factores y 36 reactivos (post análisis de componentes principales con rotación Varimax y rotación Equa$\max$ ). Cuatro factores se relacionan con empatía y uno con respeto. ${ }^{9}$
Para identificar el peso del estado emocional del paciente como variable relacionada con la interacción personal, se aplicó la escala de ansiedad y depresión hospitalaria (HAD, por sus siglas en inglés), diseñado por Zigmond y Snaith, para evaluar a pacientes físicamente enfermos hospitalizados, con 14 reactivos, siete para ansiedad y siete para depresión (mediante ponderaciones de 0 a 3). Este instrumento fue utilizado y validado en México por Robles, ${ }^{38}$ quien reportó coeficientes de consistencia interna (alpha de Cronbach) de 0.81 para ansiedad y 0.82 para depresión, con un coeficiente de correlación de la subescala de ansiedad con la Escala de ansiedad rasgo estado de 0.71 y un coeficiente de correlación de la subescala de depresión con el Inventario de depresión de Beck de 0.74. En ellos, la mayor cantidad de puntos alcanzables es de 21 y las puntuaciones mayores de 10 indican morbilidad. Puntuaciones de entre ocho y 10 se interpretan como limítrofes y las inferiores a ocho como normales.

Los datos se analizaron mediante los siguientes procedimientos: dendogramas de clusters para identificar las agrupaciones de los reactivos, correlaciones de Spearman para obtener la validez concurrente entre instrumentos y análisis factorial, a fin de sustentar la reducción de reactivos y validez de constructo; todo con el apoyo de los programas R Psych y SPSS Versión 15 para Windows P.C.

\section{RESULTADOS}

Descripción de la muestra: el promedio de edad del grupo estudiado correspondió a 45.1 (D.S. 14.46), con una moda de 48. Respecto al género, $57.3 \%$ eran mujeres y el resto hombres. En cuanto al estado civil, $77.7 \%$ eran casados y casi una tercera parte de los pacientes (29.6\%) sólo tenían estudios de primaria completa o incompleta, otro $33.8 \%$, con secundaria completa o incompleta. El $20.7 \%$ tenía preparatoria o estudios técnico-profesionales y un $15.9 \%$, estudios de licenciatura o más avanzados.

El instrumento CECOP se constituyó con 25 reactivos que representan conductas observables, los cuales se contestan en una escala de cinco opciones de respuesta tipo Likert, que van desde siempre hasta nunca. Los comportamientos observados por los pacientes en sus enfermeras durante la interacción incluyeron algunos reactivos sencillos sobre una sola conducta no verbal, como sonreír. Otros reactivos se refirieron a comportamientos relativamente complejos, compuestos de conductas verbales y no verbales, como me apapacha.

Las pruebas subsecuentes aplicadas a 13 pacientes en el segundo piloteo sugirieron la modificación de la redacción en algunos de los reactivos. A manera de ejemplos, en la primera prueba había frases textuales como: a) "lo critican", que se modificó para quedar "respetan su forma de pensar"; b) "le dan órdenes sin darle explicaciones", que se cambió 
Cuadro 1. Instrumento: CECOP (23 reactivos)

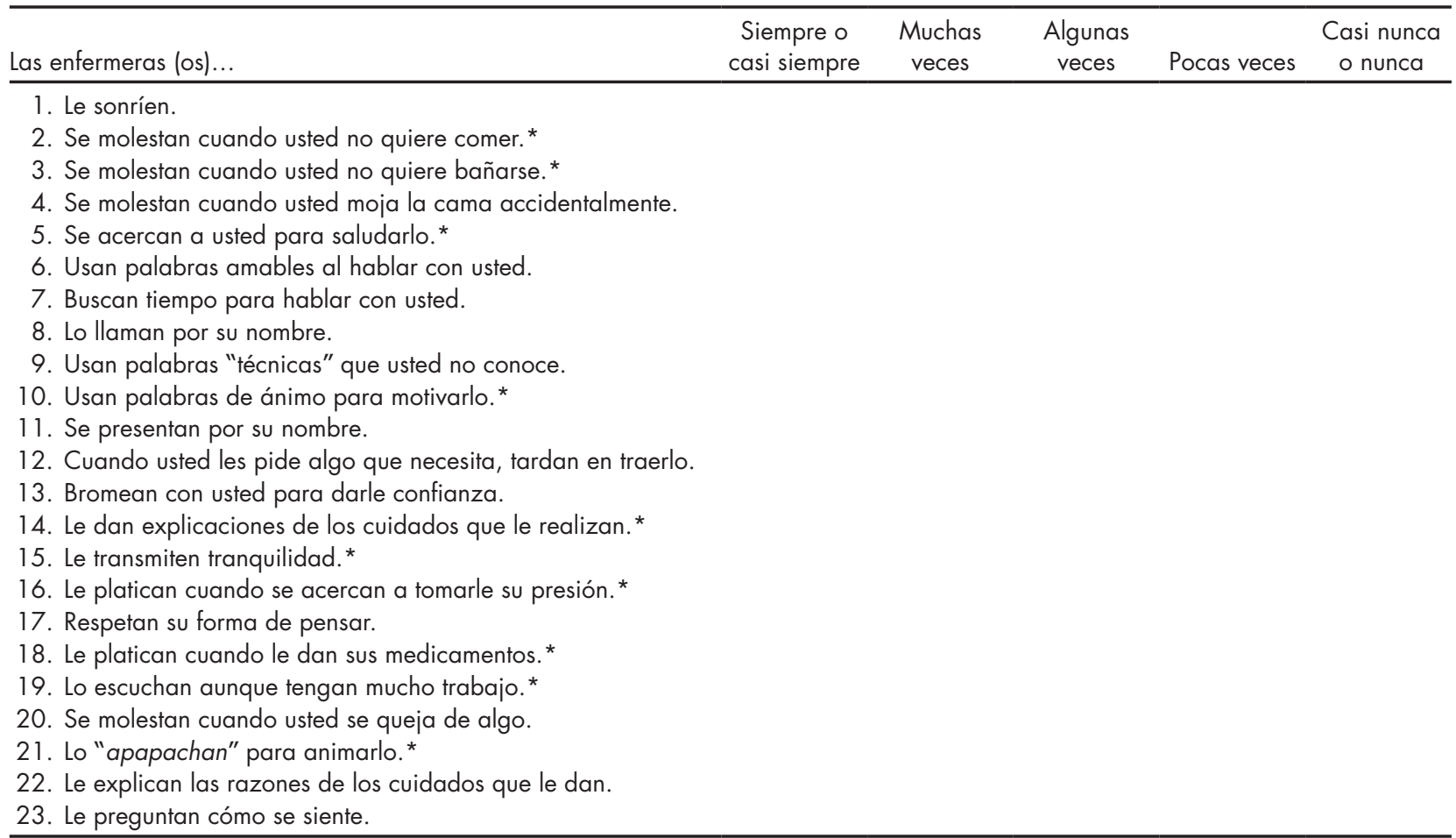

* Reactivos que permanecen en el CECOP breve (10 reactivos).

por "le dan explicaciones de los cuidados que le realizan" y c) "lo regañan cuando usted no quiere comer", que se transformó en "se molestan cuando usted no quiere comer".

Las respuestas de los jueces expertos se analizaron con el coeficiente de VCG, el cual resultó en .83, y con el coeficiente de VCR por reactivo, que osciló entre .50 y 1.00 . Conforme al criterio sugerido por Tristán, ${ }^{37}$ según el cual los reactivos calificados con coeficientes inferiores a .58 se eliminan, se excluyeron dos reactivos: "manifiestan malestar al tener que tocar directamente su piel" y "se ven inquietas cuando tienen mucho trabajo", que arrojaron valores de .50.

Se conservaron 23 reactivos; su VCG fue de .86. Los reactivos mantuvieron características heterogéneas en cuanto a la sencillez o complejidad, según la clasificación que hace Caballo $^{39}$ respecto a instrumentos que miden habilidades sociales.

Después de la validación de contenido por expertos, se procedió a la prueba del instrumento para establecer su validez de constructo mediante su aplicación a 150 pacientes hospitalizados (cuadro 1).

Se utilizó el análisis de cúmulos o racimos (clusters) para identificar la agrupación de los reactivos, conservando la congruencia conceptual del mismo. Se procedió a la eliminación progresiva de aquellos reactivos que cayeron en alguno de los siguientes criterios: con cargas factoriales menores a .50 , aislados en los clusters, con correlaciones inferiores a .40 y con VCR inferior a .83 (figura 1 ).

Se conservaron 10 reactivos, considerando siempre su estructura y aspecto conceptual..$^{24,30,32}$ Posteriormente se realizó un análisis factorial para examinar la estructura del instrumento con el método de extracción de análisis de componentes principales con rotación Varimax. El valor determinante de la matriz de correlaciones fue de .30, $\mathrm{p} \leq .001$, lo que indica que las variables funcionan relacionadas. La medida de adecuación muestral KMO fue de .791, p $\leq .001$, que indica que hay homogeneidad entre las variables de cada factor y heterogeneidad entre las variables de otros factores. Por último, la prueba de Bartlett mostró también una significancia

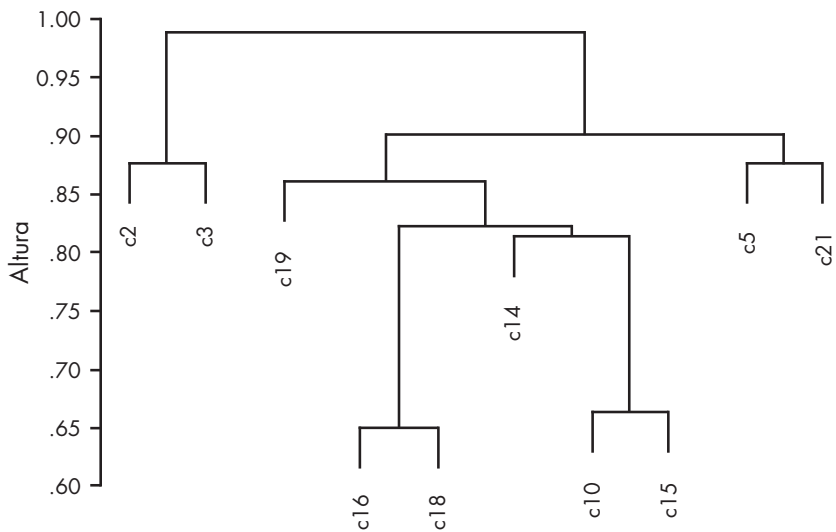

Figura 1. Dendograma. Imagen de la forma de agrupación de los reactivos que integra el instrumento de 23 reactivos; el c2 y el c3 corresponden al factor respeto y el resto a empatía. Los reactivos ausentes no formaron clusters. 
Cuadro 2. Cargas factoriales de los reactivos retenidos

\begin{tabular}{lc}
\hline Factor & Carga factorial \\
\hline Factor 1 Empatía & \\
Reactivo 16 & 0.748 \\
Reactivo 15 & 0.703 \\
Reactivo 10 & 0.689 \\
Reactivo 18 & 0.671 \\
Reactivo 14 & 0.565 \\
Reactivo 19 & 0.540 \\
Reactivo 5 & 0.516 \\
Reactivo 21 & 0.512 \\
Factor 2 Respeto & \\
Reactivo 2 & 0.825 \\
Reactivo 3 & 0.810 \\
\hline
\end{tabular}

estadística ( $\mathrm{p} \leq .001)$, lo que revela que el modelo factorial es adecuado para explicar los datos.

Los resultados obtenidos arrojaron dos factores con una varianza explicada de $54.58 \%$. El primero, con ocho reactivos relacionados con la empatía que explora la proximidad física, la comunicación durante la instrumentación de procedimientos técnicos (como el registro de constantes vitales y la administración de medicamentos), la escucha, la explicación de los cuidados impartidos y la transmisión de ánimo, confianza y afecto por parte de la enfermera hacia el paciente.

El segundo factor quedó constituido por dos reactivos relacionados con el respeto que exploran el comportamiento de la enfermera ante la resistencia del paciente para el cumplimiento de hábitos relacionados con las funciones biológicas, como la alimentación y el baño. Aunque este factor sólo tuvo dos reactivos, su carga factorial fue muy alta y mostró identidad independiente (cuadro 2).

El instrumento incluyó tres preguntas más que indagan la apreciación del paciente con respecto a la comprensión y el respeto por parte de las enfermeras. Dichos aspectos se calificaron con escalas análogo-visuales de uno a 10. También se midió con una escala similar el grado de satisfacción experimentada por el paciente cuando recibía el cuidado de las enfermeras durante su estancia hospitalaria.

Se examinó la validación convergente en las dos versiones del CECOP, la extensa (de 23 reactivos) y la breve (de 10 reactivos) a fin de conservar ambos instrumentos para el desarrollo de estudios posteriores con poblaciones más amplias y diversas.

La validación convergente por medio de la correlación de puntajes totales del CECOP (con 23 reactivos) y el CAT (2007) que evalúa interacción personal, mostró una correlación de Spearman de .544 (ps.001). La correlación entre los puntajes totales del CECOP y la escala análogo visual sobre comprensión fue de 457 ( $\mathrm{p} \leq .001)$, mientras que la correlación de los puntajes del CECOP con la escala de respeto fue de .277 ( $\mathrm{p} \leq .001)$. Por último, la correlación del CECOP-23 con la escala de satisfacción fue de .520 (ps.001). No fue po- sible establecer la correlación divergente entre el CECOP y el HADS debido a la baja prevalencia de ambos factores en el grupo de estudio.

La validación convergente mediante la correlación del CECOP en su versión breve con el CAT fue de .459 (ps.001). La correlación del CECOP-10 con la escala análogo visual de comprensión fue de 419 ( $\mathrm{p} \leq .001)$. No hubo correlación significativa con la escala de respeto. La correlación del CECOP-10 con la escala análogo visual de satisfacción fue de .495 ( $\mathrm{p} \leq .001)$.

El nivel de consistencia interna del CECOP extenso (23 reactivos) fue de .812; la escala reducida a 10 reactivos conservó un alto coeficiente alfa de Cronbach (.801); los reactivos del factor 1 (empatía) mostraron un alfa de Cronbach de .817 y los del factor 2 (respeto), de .780 .

\section{DISCUSIÓN Y CONCLUSIÓN}

El diseño y la validación de un instrumento surgido de la exploración con pacientes hospitalizados ofrecen una herramienta que permitirá realizar mediciones más afines a la realidad nacional, en tanto que proviene de la observación del paciente sobre el comportamiento de las enfermeras y el efecto que le genera como persona hospitalizada.

La selección de los constructos empatía y respeto se sustenta en la tesis de Cormier y Cormier, ${ }^{30}$ quienes consideran que estos dos elementos son facilitadores de la relación terapéutica. Si bien la tarea de la enfermera se centra en los cuidados asistenciales dirigidos a las necesidades de carácter predominantemente biológico y en los cuidados técnicos derivados de las alteraciones que ocasionan los padecimientos, ambos se respaldan en los cuidados humanos o de relación interpersonal.

Aun cuando hubo diferencias entre los valores de las correlaciones establecidas con el CECOP breve y el extenso, al igual que con el alfa de Cronbach, las diferencias son pequeñas y la versión breve posee una mejor estructura factorial. Además, ofrece ventajas relacionadas con su extensión, ya que puede ser aplicada rápidamente en estudios de investigación con muestras amplias o en las que requieren evaluarse otras variables.

La interacción con el paciente es fundamental en el desempeño de la profesión y constituye el núcleo de la aplicación de los cuidados al integrar los elementos biológicos, técnicos y asistenciales. ${ }^{26}$ También es fuente de información para identificar las necesidades emergentes o no contempladas y permite evaluar la ayuda que se requiere para individualizar la aplicación de los cuidados asistenciales y técnicos. ${ }^{1,15}$

Estos hallazgos surgen en un contexto en que la mayoría de los instrumentos son traducciones o muestran poca sensibilidad a las condiciones relevantes para el paciente hospitalizado en México, por lo que suelen mostrar efectos de techo en la evaluación de los servicios de salud. ${ }^{29,40}$ Durante el presen- 
te estudio, cuando se utilizó la traducción del instrumento de Duffy, ${ }^{9}$ por ejemplo, se observó cansancio en los participantes y dificultad para comprender algunos reactivos.

El procedimiento de entrevista a pacientes durante su hospitalización y la validación de expertos permitieron la inclusión de reactivos que abarcan elementos de calidad en la relación interpersonal como indicadores de comprensión y respeto, tanto desde el punto de vista teórico como a partir del lenguaje y percepción de los pacientes mexicanos. Lo anterior fue posible al insertar precisiones en la redacción de los reactivos con palabras expresadas por los propios pacientes. Cabe señalar que, en algunos casos, los pacientes mostraron dificultad para reconocer que podría haber actitudes de las enfermeras no favorables hacia ellos, hecho que sugiere predominancia de experiencias positivas, influencia de sus expectativas o deseabilidad social. ${ }^{41}$

La obtención final de dos factores fue gradual y escalonada, y estuvo sustentada en diversas pruebas estadísticas. Los factores obtenidos mostraron congruencia con los conceptos seleccionados ${ }^{24,30}$ y con la validación de contenido por los jueces expertos, a excepción de un reactivo. Éste quedó clasificado como respeto y el análisis factorial derivado de las 150 encuestas a pacientes hospitalizados lo clasificó en el rubro de comprensión empática. Esta diferencia puede explicarse por la interacción que se da entre los dos elementos facilitadores de la relación terapéutica. ${ }^{30}$

Los ocho reactivos conservados en el factor 1 (empatía), fueron correlacionados de forma positiva con la valoración general de la escala análoga visual sobre la comprensión y con la de satisfacción del paciente. Dicha validez convergente resulta otro parámetro que apoya la calidad en la medición de la relación interpersonal por medio del CECOP.

Los dos reactivos agrupados en el factor 2, relativos al respeto o aceptación positiva, arrojaron cargas importantes, mostraron correlaciones altas y se aislaron claramente en el dendograma. Sin embargo, fue modesta su correlación con la valoración de la escala análoga visual sobre el respeto.

Lo anterior denota la necesidad de buscar más comportamientos claramente reconocibles como expresión de respeto. En contraparte, los reactivos de empatía fueron variados e identificables, lo que puede denotar una diferencia cultural importante en su expresión en México. Por esta razón, los instrumentos diseñados en otras culturas suelen mostrar insensibilidad o resultar poco claros.

La correlación positiva entre los puntajes totales del CECOP y del CAT, ${ }^{9}$ aplicados simultáneamente a los 150 pacientes, muestra otra faceta positiva de la validación. Ambos instrumentos se diseñaron para medir interacción enfermera-paciente. En el caso del CAT, en sus primeras aplicaciones se obtuvieron ocho factores en 36 reactivos, ${ }^{9} \mathrm{de}$ los cuales cuatro se referían a comprensión empática y uno a respeto. En estudios posteriores, se conservaron 26 reactivos y se encontró un solo factor identificado genéricamente como interacción enfermera-paciente. ${ }^{10}$
La aplicación del CAT en México, previa autorización de la autora, se apegó a las recomendaciones de la Organización Mundial de la Salud sobre procedimientos de adaptación y traducción de instrumentos. Si bien esa aplicación en particular no respondía completamente al propósito central del presente estudio, sí había una medición paralela de algunos constructos entre el CAT y el CECOP. En ambos instrumentos, la mayoría de los pacientes tienden a calificar positivamente la interacción de las enfermeras.

A partir del análisis factorial aplicado al CECOP, con 23 reactivos distribuidos en las dimensiones de comprensión y respeto, se observó una mayor retención de los orientados a la comprensión empática, concebiblemente más relevante, o al menos más identificable. Resalta así la importancia del entrenamiento de las enfermeras en habilidades para la comunicación como la escucha, la respuesta a sentimientos del otro, la expresión de afectos y la expresión de aliento o ánimo, entre otras. ${ }^{42}$ Las habilidades son susceptibles de mejora mediante programas de entrenamiento y sensibilización, y se espera que al ser practicadas por las enfermeras capacitadas muestren incremento en los puntajes obtenidos en el CECOP.

Como limitación principal, se reconoce que el CECOP, aun cuando ya ofrece valores útiles, requiere de aplicación en muestras más amplias y diversas a fin de aumentar su utilidad. También será importante la consideración de nuevos reactivos que brinden mayor solidez a la evaluación del factor respeto.

La empatía fue el factor más notable en esta población. Culturalmente, se trata de comportamientos relevantes para el cuidado asistencial en mexicanos y en pacientes de culturas similares y con características análogas a la muestra del estudio. Destacó la consistencia en términos culturalmente arraigados como apapacho, que no se observa en otros instrumentos.

Así, es posible la evaluación de los cuidados profesionales en su dimensión humana desde el punto de vista de los pacientes, a fin de identificar elementos susceptibles de mejora e intervención que provean condiciones de recuperación clínica, estimulada por la impartición de cuidados humanos que brinden confort y favorezcan la comunicación entre los pacientes y las enfermeras.

\section{Financiamiento}

Ninguno.

\section{Conflicto de intereses}

Los autores declararon no tener conflicto de intereses.

\section{Agradecimientos}

Agradecemos a las maestras María del Carmen Hernández Guillén y Sandra Olvera Arreola por su apoyo invaluable en el desarrollo en esta etapa de la presente investigación. 


\section{REFERENCIAS}

1. Colliere MF. Promover la vida. De la práctica de las mujeres cuidadoras a los cuidados de enfermería. Madrid, España: Interamericana McGraw-Hill; 1993.

2. Beltrán-Salazar O. La práctica de enfermería en cuidado intensivo. Aquichán 2008;8(1). Recuperado el 15 de marzo de 2011 En: http:// aquichan.unisabana.edu.com/index.php/aquichan/article/viewArticle/123/247

3. Mok E,Chiu PC. Nurse-patient relationship in palliative care. J Advanced Nursing 2004;48(5):475-483.

4. McCabe C. Nurse-patient communication: an exploration of patients' experiences. J Clinical Nursing 2004;13(1):41-49.

5. Williams AM, Irurita VF. Therapeutic an non-therapeutic interpersonal interactions: the patient's perspective. J Clinical Nursing 2004;13(7):806-815.

6. Wyatt D. How do participants of a post registration oncology nursing course perceive that course influences their practice? A descriptive survey. Eur J Oncol Nurs 2007;11(2):168-178.

7. Cossette S, Coté JK, Pepin J, Ricard N et al. A dimensional structure of nurse-patient interactions from a care perspective: refinement of the Caring Nurse-Patient Interaction Scale (CNPI-Short Scale). J Advanced Nursing 2006;55(2):198-214.

8. Cossette S, Pepin J, Coté JK, Courval FP. The multidimensionality of caring: a confirmatory factor analysis of the Caring Nurse Patient Interaction Short Scale. J Advanced Nursing 2008;61(6):699-710.

9. Duffy JR, Hoskins L, Furst-Seifert R. Dimensions of caring. Psychometric evaluation the caring assessment tool. Advances Nursing Science 2007;30(3):235-245.

10. Duffy JR, Brewer BB, Weaver MT. Revision and psychometric properties of the caring assessment tool. Clinical Nursing Research 2010;20(10):1-14.

11. Thomas LH, McColl E, Priest J, Bond S et al. Newcastle satisfaction with nursing scales: an instrument for quality assessments of nursing care. Quality Health Care 1996; 5:67-72.

12. Sierra MT, Cardona LP, Bernal MF, Forero AE. Percepción del cuidado de enfermería en diferentes instituciones hospitalarias de la ciudad de Bogotá. Avances Enfermería 2006;24(1):68-72.

13. Williams AM, Kristianson LJ. Emotional care experienced by hospitalised patients: development and testing of a measurement instrument. J Clinical Nursing 2009;18(7):1069-1077.

14. Bowles N, Mackintosh C, Torn A. Nurses' communication skills: an evaluating of the impact of solution-focused communication training. J Advanced Nursing 2001;36(3):347-354.

15. De los Ríos JL, Sánchez-Sosa JJ. Well-being and medical recovery in the critical care unit: the role of the nurse-patient interaction. Salud Mental 2002;25(2):21-31.

16. McGilton K, Brien-Pallas LL, Darlington G, Evans M et al. Effects of relationship-enhancing program of care on outcomes. J Nursing Scholarship 2003;35(2):151-156.

17. McGilton K, Irwin-Robinson H, Boscart V, Spanjevic L. Communication enhancement: nurse and patient satisfaction outcomes in a complex continuing care facility. J Advanced Nursing 2006;54(1):35-44.

18. Wilkinson S, Perry R, Blanchard K, Linsell L. Effectiveness of threeday communication skills course in changing nurses' communication skills with cancer/palliative care patients: a randomized controlled trial. Palliative Medicine 2008;22:365-375.

19. Mullan B, Kothe EJ. Evaluating a nursing communication skills training course: The relationships between self-rated ability, satisfaction, and actual performance. Nurse Education Practice 2010;10:374-378.
20. Nava MG, Zamora P. Percepción del trato digno de los familiares de los enfermos neurológicos en el Instituto Nacional de Neurología y Neurocirugía. Enfermería Neurológica 2010;9(1):32-38.

21. Nava-Gómez M, Brito-Ortiz JF, Valdez-Escobedo A, Juárez-García A. Trato digno otorgado por enfermeras en centros de salud en Morelos, México. Rev Enferm Inst Mex Seguro Soc 2011;19(2):91-98.

22. Arnold EC, Underman K. Interpersonal relationships professional communication skills for nurses. St. Louis Missouri: Elsevier Saunders; 2011.

23. Balzer J. Communication in nursing. St. Louis Missouri: Mosby Elsevier; 2008.

24. Cibanal L, Arce MC, Carballal MC. Técnicas de comunicación y relación de ayuda en ciencias de la salud. Barcelona, España: Elsevier; 2010.

25. Niven N. Psicología en enfermería. México: El Manual Moderno; 2009.

26. Watson J. Nursing: Human science and human care. Norwalk, CT: Appleton-Century-Crofts; 1985.

27. Duffy JR. Caring assessment tools and the CAT-Admin. En: Watson J (ed.). Assessing and measuring caring in nursing and health science. Nueva York: Springer Publishing Company; 2009.

28. López F, Torres A. Categorización del comportamiento en la investigación observacional: Historia de un caso. Revista Mexicana Análisis Conducta 1991;17(3):7-21.

29. Ortega $C$ et al. Manual de evaluación del servicio de enfermería. Estrategias para su aplicación. México: Panamericana; 2006.

30. Cormier WH, Cormier S. Estrategias de entrevista para terapeutas. Habilidades básicas e intervenciones cognitivo-conductuales. Bilbao, España: Descleé de Brouwer S.A.; 2000.

31. Tazón Ansola P, Aseguinolaza-Chopitea L, García-Campayo J. Enfermería ciencias psicosociales. Barcelona, España: Masson; 2000.

32. Beitman BD, Yue D. Psicoterapia. Programa de formación. España: Masson; 2004.

33. Ibarra-Mendoza $X$, Siles-González J. Rol de enfermo crónico: una reflexión crítica desde la perspectiva de los cuidados enfermeros. Cultura de los cuidados. Revista Enfermería Humanidades (2006);20:129-135.

34. Quintero MT, Gómez M. El cuidado de enfermería significa ayuda. Aquichán 2010;10(1):1-12.

35. Krueger RA, Casey MA. Focus groups: A practical guide for applied research. Cuarta edición. Thousand, Oaks: Sage Publications; 2009.

36. Lawshe $\mathrm{CH}$. A quantitative approach to content validity. Personnel Psychology 1975;28(4):563-575.

37. Tristán-López A. Modificación al modelo de Lawshe para el dictamen cuantitativo de la validez de contenido de un instrumento objetivo. Avances Medición 2008;6:37-48.

38. Robles R, Morales M, Jiménez LM, Morales J. Depresión y ansiedad en mujeres con cáncer de mama: el papel de la afectividad y el soporte social. Psicooncología 2009;6(1):191-201.

39. Caballo VE. Manual de evaluación y entrenamiento de las habilidades sociales. Madrid, España: Siglo XXI; 2002.

40. Frenk J, Ruelas E, Lara R, Arroyo G et al. Evaluación de la calidad de los servicios de enfermería. Tres indicadores de aplicación hospitalaria. México: Secretaría de Salud. Subsecretaría de Innovación y Calidad, Dirección General de Calidad. Comisión Interinstitucional y Educación en Salud de Enfermería; 2003.

41. Lara-Cantú MA. Validez y confiabilidad de la escala de Deseabilidad Social de Marlowe y Crowne en una población de adultos. Salud Mental 1990;13(4):35-38.

42. Gershaw NJ, Soprafkin RP, Goldstein AP. Sé lo que anda mal...no se qué hacer. México: Diana; 1993. 